Title:

\title{
Large scale high-resolution micro-XRF analysis of histological structures in the skin of the pigeon beak
}

\section{Authors:}

Gerald Falkenberg ${ }^{1}$, Gerta Fleissner ${ }^{2}$, Guenther Fleissner ${ }^{2}$, Philipp Alraun ${ }^{1}$, Ulrike Boesenberg ${ }^{1}$, Kathryn Spiers $^{1}$

${ }^{1}$ Deutsches Elektronen-Synchrotron DESY, Photon Science, Notkestr.85, D22607 Hamburg, Germany

${ }^{2}$ Institute Cell Biology and Neurosciences, Goethe University Frankfurt/M., c/o Morphisto, Weismuellerstr. 45, D-60314 Frankfurt am Main, Germany

Corresponding author:

Dr. Gerald Falkenberg

Deutsches Elektronen-Synchrotron DESY, Hamburg, Notkestr. 85, D-22607

Hamburg, Germany

Tel.: ++49 (0)40-89982933

email: gerald.falkenberg@desy.de

\section{$\underline{\text { Keywords }}$}

pigeon beak, Columba livia, histology, Maia X-ray fluorescence detector, microscopic XRF

Short title:

Micro-XRF analysis of histological structures in the pigeon beak

Abstract:

Histological analysis of biological structures in the avian beak is performed by means of large scale high-resolution micro $x$-ray fluorescence analysis using a Maia detector system. Element maps of $\mathrm{S}, \mathrm{K}, \mathrm{Ca}, \mathrm{Fe}$ and $\mathrm{Zn}$ of square millimeter sizes show distinct cellular and subcellular tissue structures in semi-thin sections with a resolution at the (sub-)micrometer scale. Non-destructive qualitative and quantitative evaluation of the respective trace elements can be performed on the tissue samples. The elemental maps are compared with conventional differential histological stainings of parallel sections. This method is discussed as a pre-examination tool for subsequent analysis of selected structural sites by diffractive and spectroscopic X-ray 
nanoprobe techniques and, after additional ultrasectioning, also by transmission electron microscopy.

\section{$\underline{\text { Introduction }}$}

In general, the histological analysis of tissue structures is performed under a light microscope or electron microscope after distinct procedures. These distinct procedures include fixation for a good state of preservation close to the states in the living body, staining or dying with a variety of chemicals, which selectively color specific organs or tissues ${ }^{[1]}$, sectioning of the specimens into thick, thin or ultrathin slices, which are then mounted on glass slides or grids for an analysis in a light or electron microscope ${ }^{[2]}$ for higher magnification.

Studies on the amount and type of trace metals in histological tissues have gained increasing interest. For example, neurodegenerative diseases are landmarked by a higher amount of iron or aluminum in certain areas of the central nervous system ${ }^{[3]}$ and heavy metals accumulate in various plant tissues as a result of pollution ${ }^{[4]}$. Furthermore, the search for sensory systems which may detect magnetic field parameters ${ }^{[5]}$ follows the assumption of magnetic material localized in nervous system structures ${ }^{[6,7,8]}$. Staining methods for the detection of these metals may be sensitive enough to find even small quantities ${ }^{[9]}$, but generally they destroy the physical and chemical ultrastructure of these metals, making further biophysical studies on the same section impossible. Unstained parallel sections may miss the feature of interest which was located by staining of the other section. In this paper we propose a different method for the identification of trace minerals and metals in their undisturbed histological context. Unstained sections are imaged by fast scanning high-resolution X-ray fluorescence analysis (XRF) ${ }^{[10]}$. XRF is a nondestructive technique and the same sample can subsequently be used for ultrastructural analysis by light microscopy (LM) or transmission electron microscopy (TEM), and also nanoscopic X-ray analyses (X-ray diffraction (XRD), X-ray absorption near edge structure (XANES), and scanning coherent diffraction imaging (Ptychography)).

Fast scanning high-resolution XRF provides semi-quantitative distribution images of histologically relevant chemical elements ${ }^{[11-16]}$. The size and resolution of these images are comparable with the results of classical histological microscopy and show 
characteristic functional organelles and structures (dermal layers, including connective tissue with, for example, lipocytes, fibrillary networks and glands; arteries, veins, capillaries and blood corpuscles; nervous axon bundles and fine dendritic processes), which can be clearly distinguished and imaged.

In the following study, we demonstrate the possibilities of fast scanning highresolution XRF with particular investigation of the proposal that the avian beak houses a still enigmatic magneto receptor system based on magnetic minerals within nervous terminals. We compare semi-quantitative XRF element maps of pigeon beak tissue with histologically stained parallel sections and describe the anatomy of the avian dermis ${ }^{[18]}$ on the millimeter scale with a sub-micrometer resolution for various skin organelles and structures. The knowledge of the element topography of the dermis is expected to be helpful in ensuing studies investigating_the location and identification of iron-mineral containing dendrites which are potentially involved in avian magnetoreception ${ }^{[5,6,8]}$. Fast scanning high-resolution XRF produces rasterscanned images of millions of data points within each single image, ensuring the technique is very well suited for the "search for the needle in the haystack", such as the mineral dendrites in the avian dermis, and relating their position to the ambient histological context.

\section{Material and methods}

\section{Fast scanning high-resolution X-ray fluorescence analysis}

The X-ray fluorescence measurements were performed at the Microprobe setup of Beamline P06 at the PETRA III storage ring at the Deutsches ElektronenSynchrotron DESY utilising a Maia X-ray fluorescence detector system. The undulator beam was monochromatized by a cryogenically cooled double crystal monochromator at $13.5 \mathrm{keV}$. The beam was focused to $400 \times 600 \mathrm{~nm}^{2}$ (hor. $\times$ ver.) using a Rh coated Kirkpatrick-Baez (KB) mirror optic yielding a flux in the focused beam of $\sim 5 \times 10^{10}$ photons $/ \mathrm{s}$. The Maia $384 \mathrm{C}$ detector, is positioned between $\mathrm{KB}$ system and sample in backscatter geometry with the incident beam passing through a hole in the centre of the detector (see Fig. 1). The detector sensor is a $500 \mu \mathrm{m}$ thick Si monolithic array of 384 detector elements of $1 \mathrm{~mm}^{2}$ area. The detector design is optimized for a target-to-detector sensor distance of $10 \mathrm{~mm}$, which corresponds to a total solid angle of $1.3 \mathrm{sr}$. The detector sensor is sealed with a $50 \mu \mathrm{m}$ thick Be window which is recessed in the detector housing. The design distance from the 
housing to the sample surface is $1.6 \mathrm{~mm}$. Scans of the $10 \mu \mathrm{m}$ thick beak dermis samples generated about 5 million counts per second in the Maia detector at moderate detector deadtime and energy resolution (<300 eV FWHM at $5.9 \mathrm{keV}$ ). Elements down to atomic number 15 (phosphorus) are detected. The integration of the detector system at the beamline P06 has been described elsewhere ${ }^{[10]}$. Accurate positioning and motion control of the sample is achieved by an encoder controlled stage system providing $50 \mathrm{~mm}$ travel range with $20 \mathrm{~nm}$ precision. The incremental encoder signals from the sample stages are fed directly into the Maia control unit to ensure accurate correlation between detector signal and sample position in continuous scanning mode. Dwell times per pixel can be as short as $0.1 \mathrm{~ms}$, but for the pigeon dermis analysis $15 \mathrm{~ms}$ exposure per scan point was used in order to provide adequate statistics for trace element analysis. For instance, an image of $1 \times 1$ $\mathrm{mm}^{2}$ size could be measured in 5 hours with $1 \mu \mathrm{m}^{2}$ resolution.

Spectral deconvolution and image frames were generated using the GeoPIXE software ${ }^{[19]}$ using a Dynamic Analysis method. A reference metal foil (Au) was measured as an external standard for quantification. Precise alignment of the sample was done under the optical microscope at the beamline, followed by a fast, low spatial resolution XRF scan from which the coordinates of the final high spatial resolution XRF scan were determined. GeoPIXE displays real-time elemental images of the sample during the scan. Quantitative analysis is done after completion of the measurement. Element maps were exported in TIFF format displaying concentration, counts or areal density loading per pixel. Final processing was performed using the program package imageJ.

\section{Experimental Animals}

Homing pigeons (Columba livia) were raised in the loft of the biological institutes of the Goethe University in Frankfurt a. M. and regularly trained for free-flight homing prior to histological investigation. The experiments were performed in accordance with the rules and regulations for animal welfare in Germany.

\section{Histological processing:}

For histological processing the animals received lethal anesthesia by a high dosage Narcoren injection followed by transcardial perfusion with $4 \%$ paraformaldehyde. 
Afterwards the inner lining of the upper beak was carefully isolated from the hard components of the beak (bone as well as horny sheath) by means of iron-free titanium and ceramic tools. The tissue was resin-embedded (Araldite), then cut with a diamond knife in $10 \mu \mathrm{m}$ thick sections (for further details see ${ }^{[6,8]}$ ). The sections were mounted in the hole of a metal free plastic tape as sample holder for the XRF measurements. For comparative differential light microscopical analysis parallel sections were mounted on glass slides. The section for the XRF-measurements remained unstained, while the parallel control sections were stained by MassonTrichrome Goldner (staining result: ceratin and muscle fibers appear red, collagen and bones blue and green, cytoplasm pink and cellular nuclei brown-black) ${ }^{[1]}$. Histological sections were analyzed and documented by a Polyvar microscope (Reichert, Vienna-Austria).

\section{$\underline{\text { Results and Discussion }}$}

In total, eight high-resolution XRF scans of several square millimeter areas from 5 sections of 3 pigeon samples were made, from which measurements of one section are presented. Selected element maps of one complete scan are shown in figure 2 to illustrate element distributions in the global context. Several skin organelles and structures are displayed in four cropped enlargements of the same scan (Fig. 4, Fig. 6-8) demonstrating the capability to digitally zoom into a large high-resolution scan. Fig. 3 and Fig. 5 are details from smaller scans recorded with higher resolution. All $\mathrm{XRF}$ images are compared with microscopy images of histologically stained parallel sections.

The analyzed section is part of the upper beak of a homing pigeon. It contains the soft inner lining, which has been isolated from the horny sheath, the ramphotheca ${ }^{[20]}$, and contains all histological components characteristic for avian dermal tissue ${ }^{[18]}$. The section sample is localized next to the choanal cleft and shows the typical layers epidermis, dermis and hypodermis. The layers are partly modified in respect of their thickness and their cellular composition in the soft palate ${ }^{[21]}$.

In overview: The different element mappings characterize different histological structures. 
The high resolution XRF analyses reveal nearly all tissue components, which have been documented in the histologically stained parallel section (Fig. 2f): the blood system including arteries, veins and blood cells, nervous axons and dendrites, glands especially the palate mucosa and different types of connective tissue. Only cellular nuclei and some types of blood cells are not visible in the XRF images.

The high resolution XRF mappings of $\mathrm{Ca}, \mathrm{Fe}, \mathrm{K}, \mathrm{S}$, and $\mathrm{Zn}$ of the avian beak tissue outline distinct histological structures (Fig. 2) and are discussed in the following.

\section{Calcium map (Fig. 2a)}

$\mathrm{Ca}$ is most highly concentrated in nervous axon bundles, with multiple thin fibers running in parallel wave forms. At several sites thin fibers occur in different length and diameter within the hypodermis, next to the basement membrane of the mucosal epithelia of the palate, near the blood vessels and as thinner components in deeper layers of the dermis. The endothel of arteries is encircled by calcium in contrast to the wall of veins and capillaries. Scattered tiny particles occur at random sites all over the section (most probably contaminants).

\section{Iron map (Fig. 2b)}

The iron mapping outlines nearly all tissue components. The blood system is clearly visible from the iron content of the erythrocytes inside the vessels. The walls of arteries and veins contain iron in their outer layer. In blood vessels of a smaller diameter these peripheral iron adherences are a clear indication of their nature as capillaries. A layer of delicate fibrous material just below the palate mucosa may contain peripheral nervous components.

\section{Potassium map (Fig 2c)}

Arteries as well as veins and capillaries can be clearly discriminated from the background by the densely filled lumen. The vessel walls are observed to contain a high amount of potassium. A thick bundle of nervous axons, discernable by the waveshaped fibers, contains significant potassium, while the smaller branches can hardly be seen. The basal rim of the cells of the palate mucosa and the mucosal gland contain a large amount of potassium in their lumen. All cell membranes contain potassium, thus the connective tissue cells can be recognized. A connective tissue 
layer just below the palate mucosa is marked more strongly than the rest of the dermis.

\section{Sulfur map (Fig. 2d)}

Sulfur occurs inside the lumen of blood vessels, especially adhering to the inner side of the walls of veins. Nervous components are low in sulfur concentration. The glands, the mucosal gland and the palisade cells of the palate mucosa contain sulfur inside their walls but not in the lumen.

\section{Zinc map (Fig. 2e)}

Blood vessels of all diameters are shown by a distinct zinc accumulation in their lumen and endothel. The palisades of the palate mucosa and the cells of the mucosal gland contain zinc. A layer just below the palisade cells contains a higher amount of zinc than the rest of the epidermis. In the nervous structures, especially in their sheath, little zinc can be detected. A few bundles of unknown histological origin have extremely high concentrations of zinc. They might be capillaries or parts of blood vessel walls, which do not show their lumen here.

\section{Parallel section stained with Trichrome-Goldner (Fig. 2f)}

Structural details of the beak tissue can be recognized: $\mathbf{v}$ veins, ar arteries, $\mathbf{n}$ nerve, gl gland, pm palate mucosa. The layers of the beak tissue are the outer epidermis and dermis (d) and sponge-like hypodermis ( $h$ ). Due to the site of the section near the choanal cleft, the epidermis is not covered by a stratum corneum with dead cells.

In detail: A comparison of the element maps of distinct histological components accentuates structural details

\section{Blood cells}

$\mathrm{Fe}$, as an essential part of hemoglobin, and also $\mathrm{Zn}$ and $\mathrm{K}$ map the erythrocytes but none of the other blood cells in our measurements (Fig. 3, bottom). These maps also demonstrate the resolution of the XRF images, where capillaries, and even scattered erythrocytes, are recognizable. The LM view of erythrocytes indicates an avian specialty: Different to the mammalian system, the avian erythrocytes have nuclei, which are clearly visible in the LM image and also in the $\mathrm{K}$ map. 
Blood vessels

Veins:

$\mathrm{Fe}, \mathrm{K}$ and $\mathrm{Zn}$ maps show the three distinct layers of the wall of veins, which are hardly discernable in the LM comparison (Fig. 3, top). The thin inner lining of the vessel, the endothel, is best visible in the $\mathrm{K}$ map. The tunica media, with circular muscle fibers, which may regulate the diameter of the vein, can be detected in $\mathrm{K}$ and $\mathrm{Zn}$ maps as tiny dots (in cross section) and as short stripes in the longitudinal view. The outer layer, membrana elastica externa, with a dense fiber network perpendicular to the tunica media contains multiple blood capillaries, which are shown in Fe and $\mathrm{Zn}$ maps.

Arteries:

The element maps reveal, more clearly than shown in the LM image, that the wall of arteries is composed of three different layers (Fig. 4): a thin inner endothel (membrana elastica, see $\mathrm{Ca}$ and $\mathrm{S}$ maps), the thick media consisting of muscle cells and elastic fibers (visible in all maps) covered by the adventitia composed of elastic and collagenous fibers. Similar to observations in the wall of veins, multiple capillaries (cf. erythrocytes) occur around the adventitia.

\section{Nervous components}

Nervous tissue is marked by its high content of $\mathrm{Ca}$ and $\mathrm{K}$ and the spectacular wave form of the axon bundles (Fig. 5). Nervous dendrites with fibres of small diameter form loose networks, which can be discriminated from the densely packed connective tissue fibres and the capillaries containing erythrocytes (Fe map, lower right)

\section{Glands}

Two types of exocrine glands, salt glands and the mucosal dermis of the palate, occur in the avian beak (Fig. 6 and see Fig 2). They have basically a similar structure: the exudate is produced in the depth of small tubules and then transported to the outside. XRF maps show the sites of these different steps of the process separately.

\section{Connective tissue}

Connective tissue serves different functions and is respectively composed of various cell types (Fig. 7 and 8). Two of those types are clearly discernable by XRF map: 
Univacuolar lipocytes with a clear membrane around an empty cell lumen are best visible in the Fe map but also in the $\mathrm{K}$ map (Fig. 7). They upholster, for example, against mechanical stress in the beak. Fibrocytes form dense networks (Fig. 8) stabilizing the shape of the tissue. They might be intermingled with underlying peripheral nervous fibers (see Ca maps). These images show the three layers of the epidermis in this avian skin: Mucosal cells, connective fiber network including nervous dendrites and the underlying adipose cells of the hypodermis.

\section{Conclusion}

Fast scanning high-resolution XRF element maps are, in size and resolution, comparable with results of classical histological microscopy. The element maps reveal within their undisturbed histological context nearly all tissue components, functional organelles and structures which have been documented in the histologically stained parallel section. Additionally, they provide semi-quantitative information about the local concentrations of histologically relevant chemical elements. The non-destructiveness of the method allows the continuation of the studies by measuring the same sample section at selected small areas with nanoscopic X-ray techniques without separate sample processing ${ }^{[8]}$. In particular, these pilot results encourage further studies of the pigeon beak. Previously, the different dimensions of gross (size of the beak about 2 to $5 \mathrm{~cm}$ ), fine (peripheral trigeminal nervous system) and ultrafine (magnetic material inside nervous terminals) analysis required separate sample preparation and tedious trials to correlate the different results ${ }^{[6,8]}$, whereas now this technique allows measurements on all relevant length scales to be performed on the same sample section. After locating a promising iron-containing structure in dendritic environment by fast scanning high resolution XRF the area of interest can be prepared for subsequent transmission electron microscopy (TEM) by the re-embedding ${ }^{[2]}$ of the measured section and production of several ultrathin sections. As the location in the coordinate system is preserved and thus the metal content of the respective electron dense compartments already known, it is not necessary to perform element analysis in the TEM again.

The presented precharacterisation method may finally help to solve the riddle of the magneto receptor in the beak. The structural candidates of the magneto receptor system can be biophysically characterized as containing magnetic material which is sensitive enough to size the environmental magnetic field parameters ${ }^{[22]}$, and is 
embedded in a stimulus conducting system ${ }^{[23]}$, which may process the information to the central nervous system. 


\section{$\underline{\text { References: }}$}

(1) M. Mulisch, U. Welsch, Romeis: Mikroskopische Technik. Springer Spektrum, 2010, pp. 556.

(2) J.J. Bozzola, L.D. Russell, Electron Microscopy: Principles and Techniques for Biologists. Jones and Bartlett Pub (Ma) 1998.

(3) S. Bohic, K. Murphy, P. Werner, P. Cloetens, M. Salomel̀, S. Murielle, J. Susini, K. Double, Analytical Chemistry, 2008, 80, 9557-9566.

(4) S. Mishra, M. Alfeld, R. Sobotka, E. Andresen, G. Falkenberg, J. Exp. Botany, 2016, 67 (15), 4639-4646.

(5) R. Wiltschko, W. Wiltschko, J. Comp. Physiol A, 2013, 199.2, 89-98.

(6) G. Fleissner, B. Stahl, P. Thalau, G. Falkenberg, Gue. Fleissner, Naturwissenschaften 2007; 94, 631-642.

(7) G. Fleissner, Gue. Fleissner, Magnetoreception. Encyclopedia of Animal Behavior, (Eds. M. D. Breed, J. Moore), Academic Press, Oxford, 2010, pp. 324335.

(8) G. Falkenberg, G. Fleissner, K. Schuchardt, M. Kuehbacher, P. Thalau, H. Mouritsen, D. Heyers, G. Wellenreuther, Gue. Fleissner, PLOS One, 2010, 5 (2) e9231 (online)

(9) J. Straehle, E. Schweda, Jander-Blasius: Lehrbuch der analytischen und präparativen anorganischen Chemie, Hirzel Verlag, Stuttgart, 2002

(10) U. Boesenberg, C.G. Ryan, R. Kirkham, D.P. Siddons, M. Alfeld, J. Garrevoet, T. Nunez, T. Claussen, T. Kracht, G. Falkenberg, J. Synchrotron Rad. 23, 2016, 1550-1560.

(11) M.J. Pushie, , I.J. Pickering, M. Korbas, M.J. Hackett, and G.N. George, Chemical reviews, 2014, 114(17), 8499-8541.

(12) S. Majumdar, J.R. Peralta-Videa, H. Castillo-Michel, J. Hong, C.M. Rico, and J.L. Gardea-Torresdey, Analytica chimica acta, 2012, 755, 1-16.

(13) S. A. James, D.E. Myers, M.D. de Jonge, S. Vogt, C.G. Ryan, B.A. Sexton, P. Hoobin, D. Paterson, D.L. Howard, S.C. Mayo, M. Altissimo, G.F. Moorhead, S.W. Wilkins, Anal Bioanal Chem, 2011, 401, 853-864.

(14) M.J. Niemiec, B. De Samber, J. Garrevoet, E. Vergucht, B. Vekemans, R. DeRycke, E. Bjoern, L. Sandblad, G. Wellenreuther, G. Falkenberg, P. Cloetens, L. Vincze, C.F. Urban, Metallomics, 2015, 7(6), 996 - 1010.

(15) M.J. Farquharson, K. Geraki, G. Falkenberg,R. Leek, A. Harris, Applied Radiation and Isotopes, 2007, 65(2), 183-188.

(16) D. Deruytter, J. Garrevoet, M. Vandegehuchte, E. Vergucht, B. De Samber, 
B. Vekemans, K. Appel, G. Falkenberg, K. Delbeke, R. Blust, K. De Schamphelaere, L. Vincze, C. Janssen, Environmental Science \& Technology, 2014, 48, 698-705.

(17) A. Roschger, J.G. Hofstaetter, B. Pemmer, N. Zoeger, P. Wobrauschek, G. Falkenberg, R. Simon, A. Berzlanovich, H.W. Thaler, P. Roschger, K. Klaushofer, C. Streli, Osteoarthritis and Cartilage, 2013, 21, 1707-1715.

(18) P. R. Stettenheim, Amer. Zool. 2000, 40, 461-477.

(19) C.G. Ryan, R. Kirkham, R.M. Hough, G. Moorhead, D.P. Siddons, M.D. de Jonge, D.J. Paterson, G. De Geronimo, D.L. Howard, J.S. Cleverley, Nucl. Instrum. Methods Phys. Res. A, (2010), 619, 37-43.

(20) B. Campbell, E. Lack, A Dictionary of Birds. T \& A D Poyser. 1985.

(21) H. Sagsoez, S. Erdogan, M. E. Akbalik, Acta Zoologica (Stockholm) 2013; 94(4) 382-391.

(22) R. Wiltschko, W. Wiltschko, Magnetic Orientation in Animals, Zoophysiology; Vol. 33, Springer Verlag Berlin, Heidelberg, New York, 1995.

(23) G. Fleissner, Gue. Fleissner, B. Stahl, G. Falkenberg, J. Ornithol. 2007, 148, 643-648.

\section{Acknowledgements}

The project was funded by several grants from DESY (Hamburg). We thank the team of Morphisto (Frankfurt a. M.) for their support during the histological sample preparation. The fruitful cooperation with Pete Siddons (BNL), Chris Ryan and Robin Kirkham (CSIRO, Australia) on the implementation and maintenance of the Maia detector system and the GeoPIXE software is highly appreciated.

\section{Figure Legends}

Figure 1. Experimental setup for fast XRF scanning microscopy at the PETRA III beamline P06. Left: The monochromatic beam is focused by KB mirrors onto the sample. X-ray fluorescence is detected with a 384 element Maia detector in backscatter geometry. Alternatively the sample can be inspected by an in-line beamline microscope system. Middle: The optimal distance of sample to detector housing is $1.6 \mathrm{~mm}$. Right: $\mathrm{Si}_{3} \mathrm{~N}_{4}$ windows are used as substrates for tissue samples. The detector Beryllium window and the sample holder are depicted as transparent in order to illustrate the sample to detector sensor geometry. 
Figure 2. Scanning micro-XRF element maps of a histological section of the pigeon beak skin. (a) to (e) Element maps of calcium, iron, potassium, sulfur and zinc. (f) light microscopic view of a parallel section stained by Goldner-Masson-Trichrom in order to identify the different tissue components (ar artery, $\mathbf{g}$ gland, $\mathbf{n}$ nerve, $\mathbf{p m}$ palate mucosa, $\mathbf{v}$ vein,) and dermal layers ( $\mathbf{d}$ dermis, $\mathbf{h}$ hypodermis). Scan range $5 \times 1.25 \mathrm{~mm}^{2}, 1.5 \times 1.5 \mu \mathrm{m}^{2}$ pixel size, $15 \mathrm{~ms}$ dwell time. Maximum element areal densities [ng/cm²]: Ca: 2000; Fe: 3200; K: 11000; S: 20000; Zn: 250.

Figure 3. Wall of a vein. Element maps of iron, potassium and zinc. Light microscopic (LM) view of a similar area in a parallel histological section. The thin inner lining of the vessel is covered by two thicker layers with fibers running perpendicular to each other. The avian erythrocytes (e) have nuclei. Outside the vein capillaries can be detected as light structures due to iron containing erythrocytes. Displayed scan range $0.150 \times 0.075 \mathrm{~mm}^{2}, 0.5 \times 0.5 \mu \mathrm{m}^{2}$ pixel size, $10 \mathrm{~ms}$ dwell time. Maximum element areal densities [ng/cm²]: Fe: 2700; K: 3000, Zn: 150.

Figure 4. Wall of an artery. Element maps of calcium (Ca), iron (Fe), and sulfur (S). $(\mathrm{Lm})$ Similar area in a parallel histological section. The three layers of the wall are clearly identifiable like in the wall of a vein (see Fig.2). In the midst of the artery clotted erythrocytes (e) can be seen. Like in the vein capillaries (c) environ the blood vessel. Detail of Fig 1 , displayed scan range $0.800 \times 0.36 \mathrm{~mm} 2,1.5 \times 1.5 \mu \mathrm{m}^{2}$ pixel size, $15 \mathrm{~ms}$ dwell time. Maximum element areal densities [ng/ $\left.\mathrm{cm}^{2}\right]$ : Ca: 2000 , Fe: 2800, S: 10000 .

Figure 5. Axon bundle with densely packed nervous fibers. Element maps of calcium $(\mathrm{Ca})$, iron $(\mathrm{Fe})$ and potassium $(\mathrm{K}) .(\mathrm{Lm})$ light microscopic view of a similar area. The nuclei of the myelin cells are only visible in the light microscope, not in the XRF images. Displayed scan range $0.240 \times 0.120 \mathrm{~mm}^{2}, 0.4 \times 0.4 \mu \mathrm{m}^{2}$ pixel size, $15 \mathrm{~ms}$ dwell time. Maximum element areal densities [ng/ $\mathrm{cm}^{2}$ ]: Ca: 700; Fe: 1200; K: 3400.

Figure 6. Cells of the palate mucosa. Element maps of iron (Fe), potassium (K), and zinc (Zn). (LM) light microscopic view of a similar area. The glandular tubules show 
various states of the secretory process in the different element scans, which cannot be followed in the light microscopic view. In the iron map, peripheral capillaries can be identified below and in between the glandular tubules. Detail of Fig 1, displayed scan range $0.400 \times 0.200 \mathrm{~mm}^{2}, 1.5 \times 1.5 \mu^{2}$ pixel size, $15 \mathrm{~ms}$ dwell time. Maximum element areal densities [ng/cm²]: Fe: 2000; K: 8000, Zn: 120.

Figure 7. Adipose tissue cells. Element maps of iron (Fe) and potassium (K). (LM) light microscopic view of a similar area. Only the cell walls of these monovacuolar fat cells can be recognized. Small protrusions at the wall mark the area of the nuclei and the perinuclear components. Detail of Fig 1, displayed scan range $0.230 \times 0.150 \mathrm{~mm}^{2}$, $1.5 \times 1.5 \mu \mathrm{m}^{2}$ pixel size, $15 \mathrm{~ms}$ dwell time. Maximum element areal densities $\left[\mathrm{ng} / \mathrm{cm}^{2}\right]$ : Fe: 900; K: 1800.

Figure 8. Epidermal layers in the palate mucosa. Element maps of calcium (Ca), iron (Fe) and zinc ( $\mathrm{Zn}$ ). (LM) light microscopic view of a similar area. Directly underneath the glandular cells is a connective tissue layer with a dense fibrillary network possibly including peripheral nervous dendrites (see $\mathrm{Ca}$ ). Below lays loose collagenic connective tissue with lipocytes. The light microscope would show further distinction only after differential stainings. Detail of Fig 1 , displayed scan range $0.250 \times 0.250$ $\mathrm{mm}^{2}, 1.5 \times 1.5 \mu \mathrm{m}^{2}$ pixel size, $15 \mathrm{~ms}$ dwell time. Maximum element areal densities [ng/cm²]: Ca: 800; Fe: 1200; Zn: 120. 